\title{
Expression of npas4 after cerebral ischemia in a MCAO rat model
}

\begin{abstract}
The expression levels of neuronal Per-Arnt-Sim domain protein 4 (Npas4) in response to ischemic stroke at different points in time were investigated in this study. Adult male SD Rats were randomly divided into eight groups as control (normal control) group, sham (sham operated) group, Middle Cerebral Artery Occlusion (MCAO) 3h group, MCAO 6h group, MCAO 12h group, MCAO 24h group, MCAO 48h group and MCAO $72 \mathrm{~h}$ group. We examined the mRNA and protein expression levels of Npas4 in the brain subjected to ischemic injury at different points in time by using western blotting, immuno histochemistry and Real time-qPCR. The expression level of Npas4 was up-regulated in the brain subjected to ischemic stroke. The up-regulation of Npas4 in response to cerebral ischemia in acute phase indicates that Npas4 may be involved in the pathophysiological mechanism of postinfarction.
\end{abstract}

Keywords: npas4, brain, MCAO, ischemic stroke
Volume I Issue 4 - 2015

\author{
Kun Zhang,' Jiajia Yan,' Li Guo,' Ping AO, ${ }^{2}$ \\ Wenqiang Chen, ${ }^{2}$ Claudia Taccheri, ${ }^{3}$ Bin $\mathrm{Li}^{3}{ }^{3}$ \\ Xiaoyun Liu' \\ 'Department of Neurology, the Second Hospital of Hebei \\ Medical University, China \\ ${ }^{2}$ Washington Institute for Health Sciences, USA \\ ${ }^{3}$ Department of Biochemistry and Molecular and Cellular \\ Biology, Georgetown University, USA
}

Correspondence: Xiaoyun Liu, Department of Neurology, The Second Hospital of Hebei Medical University, China, Tel +8613191887318, Fax 8882483568, Email audrey-I@|63.com

Received: October 26, 2015 | Published: November 9, 2015
Abbreviations: Npas4, neuronal per-arnt-sim (pas) domain protein 4; MCAO, middle cerebral artery occlusion

\section{Introduction}

Ischemic stroke is a worldwide public health issue. ${ }^{1}$ Diversification therapy shows partial effectiveness based on the experimental and clinical data, such as thrombolytic therapy, antiplatelet therapy, anticoagulation therapy and neuroprotection. However, nonrenewability of neurons and complexity of the nervous system render stroke morbidity and mortality rates high. Based on the current view, multiple factors are involved in the pathophysiological process of ischemia stroke, such as excitotoxicity, oxidative stress, inflammation, and apoptosis. ${ }^{2}$ These findings motivate researchers to into and ischemic neuronal damage. ${ }^{3-5}$ Previous reports show that the first $24 \mathrm{~h}$ following the ischemic insult is the critical course for the rapid neuronal death, and the multidimensional gene expression changes can be observed following experimental stroke. ${ }^{6}$ We focused on neuronal Per-Arnt-Sim (PAS) domain protein 4, which is referred to as Npas4, also known as neuronal transcription factor (NXF). Npas4 has been shown to be associated with transcriptional regulatory factors, which are important for many physiological, developmental and pathological events. ${ }^{7}$ A recent study suggests that the effects of Npas4 on the inhibition of excitatory synaptic activity are associated with neurological disorders, such as autism, schizophrenia and epilepsy. ${ }^{8}$ Here we examined the correlation between Npas4 expression and neuronal injury induced by ischemic stroke based on a middle cerebral artery occlusion model in rats. This may provide new insights into the molecular basis of ischemic stroke.

\section{Materials and methods}

\section{Animals}

Adult male Sprague-Dawley rats, purchased from Hebei Medical University Experimental Animal Center, weighting between 250 and $280 \mathrm{~g}$ were used. All rats were maintained on a $12 \mathrm{~h}$-light $/ 12 \mathrm{~h}$-dark conditioned environment, with humidity of $55 \pm 5 \%$, and all rats were free access to food and water. Rats were randomly divided into eight groups as control (normal control) group, sham (sham operated) group, MCAO 3h group, MCAO 6h group, MCAO 12h group, MCAO24h group, MCAO 48h group, MCAO 72h group. Behavior measurements were carried out at $3 \mathrm{~h}, 6 \mathrm{~h}, 12 \mathrm{~h}, 24 \mathrm{~h}, 48 \mathrm{~h}$ and $72 \mathrm{~h}$ after MCAO surgery by neural deficit scores, and then the brain samples were collected from the rats after decapitation.

\section{Permanent middle cerebral artery occlusion}

As previously described, rats were performed with permanent middle cerebral artery occlusion. ${ }^{9,10}$ Briefly speaking, rats were anesthetized by intraperitoneal injection with $10 \%$ chloral hydrate $(250 \mathrm{mg} / \mathrm{kg})$. A 2 to $2.5 \mathrm{~cm}$ incision was made in the center of neck. After CCA and ECA were ligatured, a nylon filament (diameter $0.265 \mathrm{~mm}$ ) was inserted intraluminally into the ICA about 18 to $20 \mathrm{~mm}$ deep. Gentamicin was used to protect against infection of the incision site. Sham group rats received the same surgery except for nylon filament insertion. During the surgery body temperatures of rats were maintained at $37 \pm 1^{\circ} \mathrm{C}$ by an electric blanket.

\section{Neurological deficit score}

The same examiner administered the neurological test, and was blinded to the experimental groups. When rats recovered consciousness from MCAO surgery, we test the neurological deficit score according to a modified scoring system that was developed by Longa et al. ${ }^{9}$ as follows:
i. No deficits
ii. Unable to fully extend the contralateral forelimb
iii. Completely cannot to extend the contralateral forelimb
iv. Gentle circling to the contralateral side
v. Serious circling
vi. Falling to the contralateral side. Higher neurological deficit score indicates more serious impairment of behavior injury. 


\section{Western blot}

The Total Protein Extraction Kit (Applygen Technologies Inc., Beijing, China) was used to obtain the protein of brain tissue following the manufacturer's protocols. Then we used BCA Protein Assay Reagent Kit (Applygen Technologies Inc., Beijing, China) to measure the protein concentration. Equivalent to $30 \mu \mathrm{g}$ total protein samples was applied to electrophoresis on $10 \%$ SDS/PAGE and then transferred onto PVDF membranes (Millipore Corporation, USA). ${ }^{11}$ After blocked with $1 \% \mathrm{BSA} / \mathrm{TPBS}$ for $1 \mathrm{~h}$ at room temperature, the membranes were incubated overnight at $4^{\circ} \mathrm{C}$ with anti-Npas4 (1:500, Santa Cruz Biotechnology) diluted in 1\% BSA/TPBS. Polyclonal mouse anti-beta actin antibody (1:3000, Bioworld Technology) was used as an internal control. Next day membranes were incubated by fluorescent labeling second antibodies (IRDye 800-conjugate rabbit anti-goat IgG or rabbit anti-mouse 1:5000 dilution; Rockland, Gilbertsville, PA, USA) for $1 \mathrm{~h}$ at room temperature. We analyzed the relative density of bands on Odyssey infrared scanner (LICOR Bioscience, Lincoln, NE, USA). The loading and transfer differences among samples were normalized with beta actin densitometric values.

\section{Immunohistochemical staining}

As previously described, we use paraffin-embedded sections to evaluate the expression of Npas 4 at $6 \mathrm{~h}$ and $12 \mathrm{~h}$ after MCAO..$^{12} \mathrm{In}$ Brief, brains of rats were fixed in $4 \%$ paraformaldehyde over $24 \mathrm{~h}$ at $4^{\circ} \mathrm{C}$, and then embedded in paraffin after dehydrated in different gradients of alcohols. Then we used Leica RM1850 Rotary Microtome (Leica Microsystem, Hesja, Germany) to cut the brain into $5 \mu \mathrm{m}$ per slice. Brain sections were blocked in 3\% normal donkey serum and then incubated with Npas4 goat polyclonal antibody (1:100, Santa Cruz Biotechnology) overnight at $4^{\circ} \mathrm{C}$. The secondary antibodies were used on the second day (Zhongshan Biology Technology Company, Beijing, China). Five visual fields of the ischemic cortex region and basal ganglia around the infarct core were selected to count mean density value under a 400x microscope.

\section{Real-time quantitative polymerase chain reaction}

According to the manufacturer's instructions, rat's brain from Bregma 1 to $-1 \mathrm{~mm}$ was rapidly dissected and total RNA was extracted by using Trizol Reagent (Invitrogen, Carlsbad, CA, USA). RNA $(2 \mu \mathrm{g})$ of each sample was used to reverse transcribed for synthesized cDNA. Primers were synthesized by Shanghai Biological Engineering Technology Company Limited. Forward and were 5'-AGCATTCCAGGCTCATCTGAA-3' and 5'-GGCGAAGTAAGTCTTGGTAGGATT-3'

for Npas4 and 5'-CCCATCTATGAGGGTTACGC-3' and 5'-TTTAATGTCACGCACGATTTC-3' for $\beta$-actin. Each sample was tested in triplicate and all samples were normalized by $\beta$-actin. Relative gene expression was measured with the $2-\Delta \Delta C \mathrm{C}$ method and then analyzed using logarithmic transformation.

\section{Data and statistical analysis}

All results are shown as mean \pm standard error. One-way ANOVA followed by Student-Newman-Keuls and LSD tests were used for multiple comparisons. Mann-Whitney U test was used for neurological deficit score. Differences with $P<0.05$ were considered statistically significant.

\section{Results}

\section{Expression of Npas4 protein level was significantly increased after ischemic insult}

Permanent middle cerebral artery occlusion model was used to simulate ischemic insult, which lead to a significant cortical and subcortical infarction (Figure 1A). Neurological deficit score was used to measure the brain function of the animal model. As shown in Figure $1 \mathrm{~B}$, there were significant differences among MCAO groups and control, sham group $(\mathrm{P}<0.05)$, which indicates that MCAO caused impairment on neural function. There was no significant difference among the model groups at the different points in time, suggesting that the preparation of the animal model was successful and reliable. Next, the levels of Npas 4 protein were measurement by western blot with whole tissue lysates collected from Bregma 1 to $-1 \mathrm{~mm}$ in rats. Our study suggested that the expression of Npas4 protein was enhanced significantly at $6 \mathrm{~h}$, and this enhancement persisted until $24 \mathrm{~h}$ after ischemic insult (Figure 2). As our results show in Figure 2A, although there was no statistical difference, the tendency of increased Npas4 expression was observed at $3 \mathrm{~h}$ following MCAO. This data indicated that Npas4 protein expression is regulated in response to the early stage of cerebral ischemia. We further verified the expression of Npas4 by immuno histochemistry in the neurons located in ischemic penumbra of brains subject to MCAO. Therefore, $6 \mathrm{~h}$ and $12 \mathrm{~h}$ of ischemic insult were selected as sampling time points to study Npas4 protein expression by immuno histochemistry according to our results of western blot. As expected, significantly increased Npas4 protein were found in cortex and basal ganglia at both $6 \mathrm{~h}$ and $12 \mathrm{~h}$ (Figure $3 \mathrm{~A}$ ) (Figure 3B), which was consistent with the results of western blot.
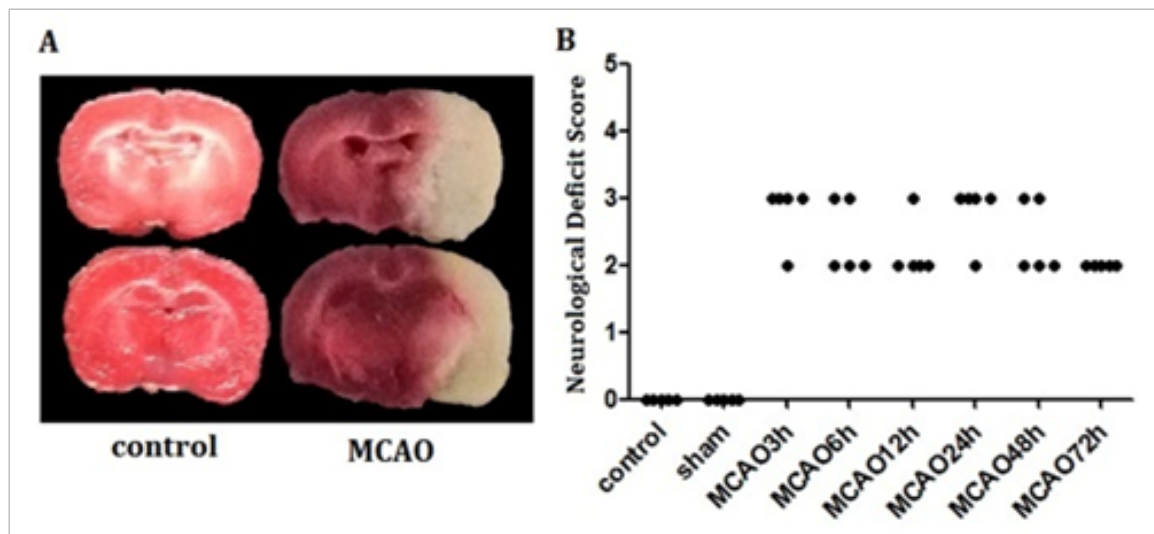

Figure I A coronal rat brain section of an animal subjected to MCAO.

A. This ischemic insult led to a significant cortical and sub cortical infarction (white) as seen with triphenyltetrazolium chloride staining.

B. Apparent neurological deficit can be observed in rats after MCAO. 


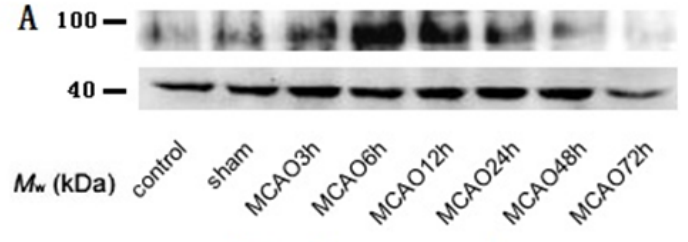

B

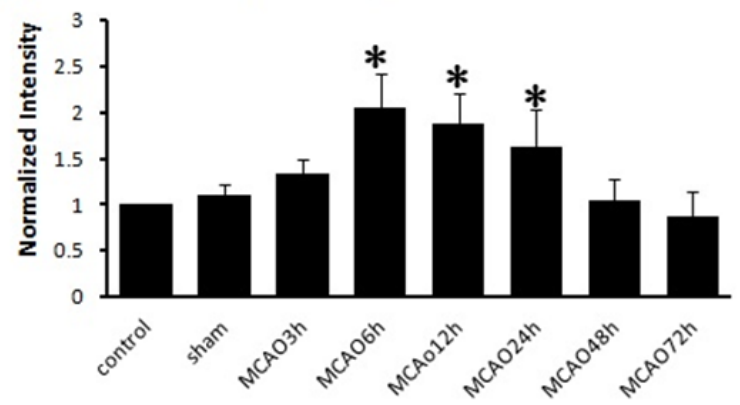

Figure 2 Western blot analysis of Npas4 at different time points after infarction. The starred group indicates Npas4 protein expression increased at 6-24hours after infraction compared with normal control group and other time points, in which the most significant increase at 6-12hours.
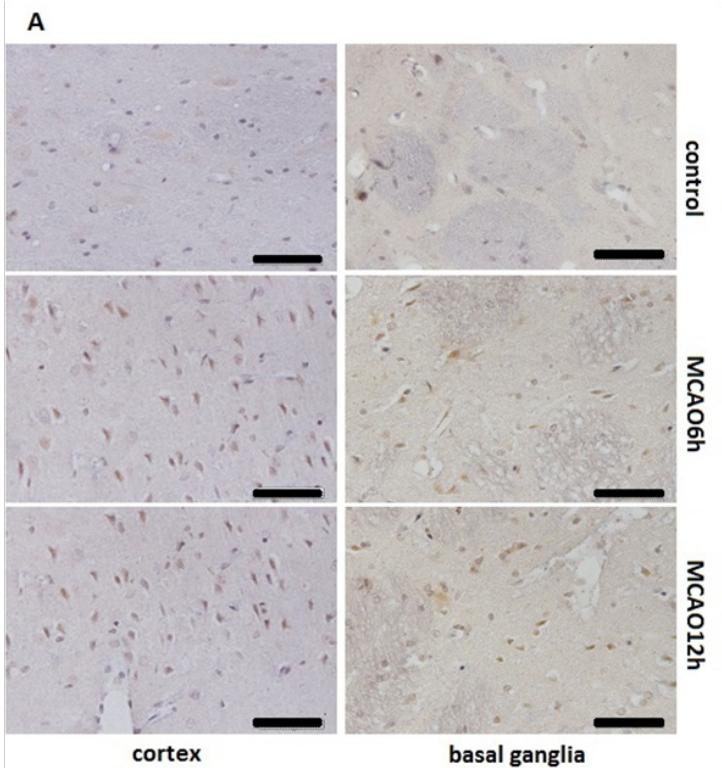

B

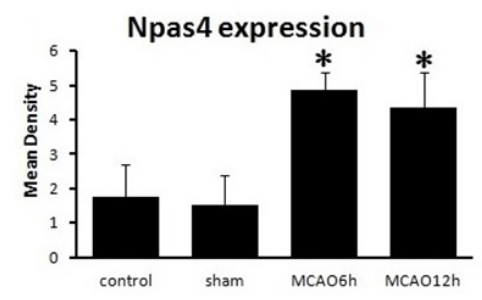

Figure 3 Immunohistochemical staining for Npas4 at $6 \mathrm{~h}$ and $12 \mathrm{~h}$ after infarction.

A. Immunohistochemical staining shows the positive cells of Npas4 were significantly increased both in cortex and basal ganglia at $6-12 \mathrm{~h}$ after MCAO.

B. The starred group suggests compared with control and sham group, the amount of positive cells of Npas 4 was significantly increased in both $6 \mathrm{~h}$ and $12 \mathrm{~h}$ after MCAO. (Scale bar $=100 \mu \mathrm{m})$.

\section{Expression of Npas4 mRNA level was significantly increased after ischemic insult}

Real-time quantitative PCR (qPCR) was used to detect the changes of Npas4 mRNA expression at different time periods following MCAO in rats as previously described. Four animal brains were studied at each time point. The results shown that Npas 4 mRNA expression gradually increased, and the significant increase was detected at $6 \mathrm{~h}-24 \mathrm{~h}$ following MCAO, then rapidly declined to normal levels after $48 \mathrm{~h}$ of MCAO (Figure 4). This result is consistent with our previous western blot experiments in the time period of Npas4 expression enhancement, which indicated that Npas4 expression was enhanced in the transcription level in response to cerebral ischemia in the early stage.

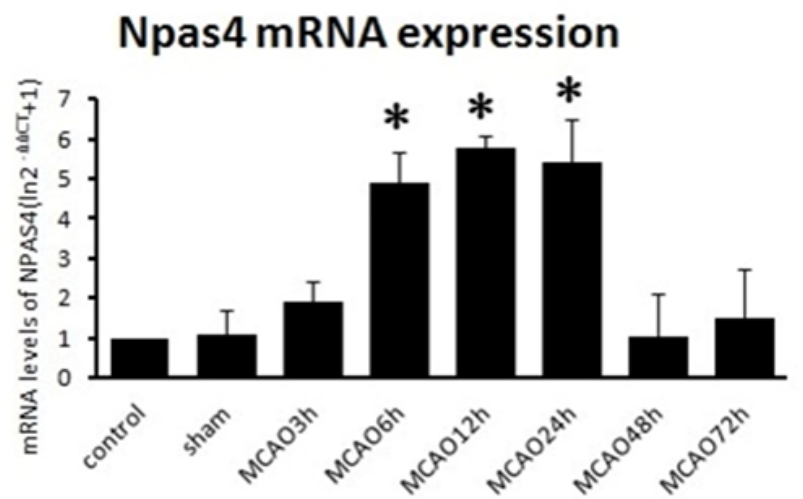

Figure 4 Real-time Quantitative PCR analysis of Npas4 at different time points after infarction. The starred group notes Npas4 Mrna expression increased at 6-24hours after MCAO compared with normal control group and other time points.

\section{Discussion}

In this study, we have described how the expression levels of Npas4 in mRNA and protein is regulated in response to ischemic insult with western blot, immuno histochemistry and Real time-qPCR technology. Our results indicated that Npas4 expression was up-regulated in brain subjected to ischemic stroke. Furthermore, this regulation was observed in the early stage of ischemic insult and the time window possibly was the first $24 \mathrm{~h}$ post-infarction. Previous studies have indicated that the Npas4 gene is regulated after ischemia during the reperfusion period when the tissue is becoming reoxygenated and ATP production is recovering. ${ }^{13}$ They also pointed out that Npas4 expression significantly increased in brain during ischemia when the tissue is extremely deprived of oxygen and ATP. ${ }^{13,14}$ Our results suggest that Npas 4 mRNA expression is significantly induced in the affected tissue of permanent occlusion, and that the significant expression initiated from first $6 \mathrm{~h}$ following MCAO remains at a high level until $24 \mathrm{~h}$. According to previous research, this prolonged expression correlates with the development of rapid neuronal damage observed in the ischemic core. ${ }^{15}$ Our data also show that expression of Npas4 protein significantly increased in the brain tissue of permanent occlusion during the time period 6-24h after MCAO. In addition, the up-regulated Npas4 protein expression was detected in both cortex and basal ganglia in rat brain exposed to ischemic insult, consistent with previous studies, which show that Npas4 expression in global cerebral ischemia. ${ }^{16}$ Our research indicates Npas4 up-regulates in response to cerebral ischemia in acute phase. This early regulation suggests that Npas4 may be involved in the pathophysiological 
mechanism of post-infarction. This result provides a novel target for further research of the pathological process and molecular mechanism of cerebral ischemic

\section{Acknowledgements}

This work was supported primarily by NIH grants to Xiaoyun Liu from the National Natural Science Foundation of China (81571160).

\section{Conflict of interest}

Author declares that there is no conflict of interest.

\section{References}

1. Donnan GA, Fisher M, Macleod M, et al. Stroke. Lancet 2008;371(9624):1612-1623.

2. Dirnagl U, Iadecola C, Moskowitz MA. Pathobiology of ischaemic stroke: an integrated view. Trends Neurosci. 1999;22(9):391-397.

3. Koistinaho J, Hokfelt T. Altered gene expression in brain ischemia. Neuroreport. 1997;8(2):i-viii.

4. Read SJ, Parsons AA, Harrison DC, et al. Stroke genomics: approaches to identify, validate, and understand ischemic stroke gene expression. $J$ Cereb Blood Flow Metab. 2001;21(7):755-778.

5. Roth A, Gill R, Certa U. Temporal and spatial gene expression patterns after experimental stroke in a rat model and characterization of PC4, a potential regulator of transcription. Mol Cell Neurosci. 2003;22(3):353-364.

6. Rickhag M, Wieloch T, Gidö G, et al. Comprehensive regional and temporal gene expression profiling of the rat brain during the first $24 \mathrm{~h}$ after experimental stroke identifies dynamic ischemia-induced gene expression patterns, and reveals a biphasic activation of genes in surviving tissue. J Neurochem. 2006;96(1):14-29.
7. Gu YZ, Hogenesch JB, Bradfield CA. The PAS superfamily: sensors of environmental and developmental signals. Annu Rev Pharmacol Toxicol. 2000;40:519-561.

8. Lin Y, Bloodgood BL, Hauser JL, et al. Activity-dependent regulation of inhibitory synapse development by Npas4. Nature. 2008;455(7217):1198-1204.

9. Longa EZ, Weinstein PR, Carlson S, et al. Reversible middle cerebral artery occlusion without craniectomy in rats. Stroke. 1989;20(1):84-91.

10. Yang $\mathrm{C}$, Zhang $\mathrm{X}$, Fan $\mathrm{H}$, et al. Curcumin upregulates transcription factor Nrf2, HO-1 expression and protects rat brains against focal ischemia. Brain Res. 2009;1282:133-141.

11. Martel MA, Ryan TJ, Bell KF, et al. The subtype of GluN2 C-terminal domain determines the response to excitotoxic insults. Neuron. 2012;74(3):543-556.

12. Qiao H, Dong $\mathrm{L}$, Zhang $\mathrm{X}$, et al. Protective effect of luteolin in experimental ischemic stroke: upregulated SOD1, CAT, Bcl-2 and claudin-5, down-regulated MDA and Bax expression. Neurochem Res. 2012;37(9):2014-2024.

13. Folbergrova J, Zhao Q, Katsura K, et al. N-tert-butyl-alphaphenylnitrone improves recovery of brain energy state in rats following transient focal ischemia. Proc Natl Acad Sci USA. 1995;92(11):5057-5061.

14. Ekholm A, Katsura K, Kristián T, et al. Coupling of cellular energy state and ion homeostasis during recovery following brain ischemia. Brain Res. 1993;604(1-2):185-191.

15. Shamloo M, Soriano L, von Schack D, et al. Npas4, a novel helix-loophelix PAS domain protein, is regulated in response to cerebral ischemia. Eur J Neurosci. 2006;24(10):2705-2720.

16. Leong WK, Klaric TS, Lin Y, et al. Upregulation of the neuronal Per-ArntSim domain protein 4 (Npas4) in the rat corticolimbic system following focal cerebral ischemia. Eur J Neurosci. 2013;37(11):1875-1884. 